\title{
Fractal and Smooth Complexities in Electroencephalographic Processing
}

\author{
Navascués MA ${ }^{1 *}$, Sebastián $\mathrm{MV}^{2}$ and Valdizán $\mathrm{JR}^{3}$
}

${ }^{1}$ Departmento de Matem'atica Aplicada, Escuela de Ingenier'ia y Arquitectura, Universidad de Zaragoza, Spain

${ }^{2}$ Centro Universitario de la Defensa, Academia General Militar, Carretera de Huesca, s/n Zaragoza, Spain

${ }^{3}$ Unidad de Trastornos del Neurodesarrollo, Clínica Montpellier, Vía de la Hispanidad, 37 Zaragoza, Spain

\begin{abstract}
The importance of the electroencephalogram (EEG) rests upon the fact that it provides useful information of the normal and pathological brain functions. However, the relations among abnormal EEG, brain functions and disorders are not well known yet. We have proposed numerical quantifiers of the EEG signal, coming from the methodology of fractal mathematics and the theory of approximation. In the first part we describe an alternative to the computation of nonlinear dimensions for this kind of signals. The approach used here is based on a fractal interpolation of the data. In the second part, we describe a method for the computation of smooth complexities based on the interpolation of EEG signals by means of polynomial splines. This kind of functions is used to find quadrature formulas for the spectral moments. Both procedures are applied to treat the electroencephalographic discrimination of a group of children suffering from an Attention Deficit with Hyperactivity Disorder (ADHD).
\end{abstract}

Keywords: Electroencephalogram; Fractal dimensions; Hjorth parameters; Attention Deficit Hyperactivity Disorder

\section{Introduction}

Hans Berger discovered in 1924 the oscillations of the electric potential of the human brain, and their trace is called electroencephalogram (EEG) since then. The importance of the EEG rests upon the fact that it provides useful information of the normal and pathological brain function. The EEG waves contain a huge quantity of information which deserves to be discovered.

All the signals with their different frequencies, amplitudes and shapes may have a value of physiological or pathological type, but the relations among abnormal EEG, brain functions and disorders are not well known yet. In many cases the discrimination of normal and pathological EEG waves is rather difficult, and the digital quantifiers may help a possible diagnosis.

The analysis of computerized EEG is an important tool nowadays in the brain sciences and psychopharmacology. The neurophysiological literature provides relevant information about the advances and applications of the field.

Our team has proposed numerical quantifiers of the EEG signal, coming from the methodology of fractal mathematics and the theory of approximation. The objective of our study consists in the determination of useful parameters characterizing the signal and its different rhythms.

Several one-single channel indices are described, along with numerical procedures for their computation. In every case, an experiment related to a disorder of attention is described. The recordings are analyzed by means of the use of the proposed quantifiers.

\section{Fractal Complexity}

A common way of facing the computation of fractal dimensions of experimental signals is the use of a phase-space model. From a single sampled signal, a whole trajectory in a higher-dimensional space is reconstructed, considering as coordinates the delays of the recording. However, this method generates a large number of algorithmic problems some of which we briefly summarize here [1].

The number of points necessary to provide a reliable estimation of the parameter can be very large [2].
The need to reach a convergence value for the dimension with respect to the number of delay variables is not fulfilled in general, as communicated in many papers about the subject $[3,4]$.

We have proposed an alternative to the computation of fractal dimensions by means of these procedures. The computation of an unknown function fitting a sampled signal can be approached by means of fractal interpolation [5,6]. A specific characteristic is the fact that the graph of these interpolants (as geometric object of the real plane) possesses a fractal dimension. This parameter constitutes a numerical index of the signal that can be used as a measure of the complexity of a variable Figure 1.

We have implemented several method of data approximation by means of fractal interpolation functions, and proved the validity and convergence of the procedures if the sampling frequency is high enough $[7,8]$.

From the point of view of the application, we look for electroencephalographic differences between normality patterns and a syndrome of lack of attention in children.

\section{Fractal interpolation functions}

In this Subsection we describe shortly the mathematical foundations of the fractal interpolation functions.

Let $K$ be a complete metric space respect the distance $d(x, y), \forall x, y$ $\in K$. Let $H$ be the set of all compact not empty subsets of $K$. Let $\forall n=1$, $2, \ldots, N, w_{n}: K \rightarrow K$, be a set of continuous maps. Then, the set $\left\{K, w_{n}\right.$;

*Corresponding author: Navascues MA, Departmento de Matem'atica Aplicada Escuela de Ingenier'ıa y Arquitectura, Universidad de Zaragoza, Spain, Tel: 34976761000; E-mail: manavas@unizar.es

Received November 06, 2014; Accepted December 31, 2014; Published January 10,2015

Citation: Navascués MA, Sebastián MV, Valdizán JR (2015) Fractal and Smooth Complexities in Electroencephalographic Processing. J Appl Computat Math 4 198. doi:10.4172/2168-9679.1000198

Copyright: @ 2015 Navascués MA, et al. This is an open-access article distributed under the terms of the Creative Commons Attribution License, which permits unrestricted use, distribution, and reproduction in any medium, provided the original author and source are credited. 


\section{FRACTAL DIMENSION}

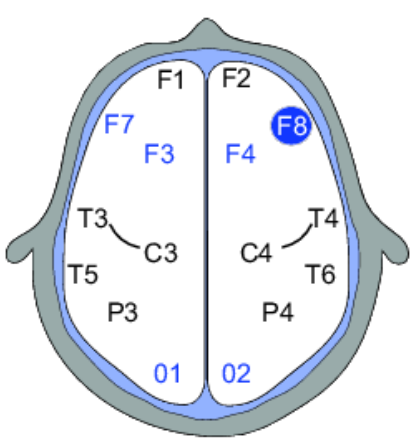

Figure 1: In blue letters,electrodes where there was no significant difference between control and ADHD children, encircled those displaying difference, in the rest the study was not performed.

$n=1,2, \ldots, N\}$ is an Iterated Function System (IFS). Define the mapping $W: H \rightarrow H$ by

$$
W(A)=\bigcup w_{n}(A) \quad \forall A \in H
$$

Any set $G \in H$ such that $W(G)=G$ is an attractor of the IFS.

Let $t_{0}<t_{1}<\ldots<t_{N}$ be real numbers, and $I=\left[t_{0}, t_{N}\right]$ the closed interval that contains them. Let a set of data points $\left\{\left(t_{n}, x_{n}\right) \in I x R: n=0,1,2, \ldots, N\right\}$ be given. Let $I_{n}=\left[t_{n-1}, t_{n}\right]$ and let $L_{n}: I \rightarrow I_{n^{\prime}}, n \in\{1,2, \ldots, N\}$, be contractive homeomorphisms such that:

$$
\begin{aligned}
& L_{n}\left(t_{0}\right)=t_{n-1}, \quad L_{n}\left(t_{N}\right)=t_{n} \\
& \left|L_{n}\left(c_{1}\right)-L_{n}\left(c_{2}\right)\right| \leq l\left|c_{1}-c_{2}\right| \quad \forall c_{1}, c_{2} \in I
\end{aligned}
$$

or some $0 \leq l<1$.

Let $-1<\alpha_{n}<1 ; n=1,2, \ldots, N, F=I x[c, d]$ for some $-\infty<c<d<\infty$ and $N$ continuous mappings, $F_{n}: F \rightarrow R$ be given satisfying:

$$
F_{n}\left(t_{0}, x_{0}\right)=x_{n-1}, F_{n}\left(t_{N}, x_{N}\right)=x_{n}
$$

where $n=1,2, \ldots, N$, and

$$
\left|F_{n}(t, x)-F_{n}(t, y)\right| \leq\left|\alpha_{n}\right||x-y|
$$

with $t \in I$, and $x, y \in R$.

Now define functions $\forall n=1,2, \ldots, N$.

$$
w_{n}(t, x)=\left(L_{n}(t), F_{n}(t, x)\right)
$$

Theorem [5,6]: The Iterated Function System (IFS) $\left\{F, w_{n}: n=1\right.$, $2, \ldots, N\}$ defined above admits a unique attractor $G$. $G$ is the graph of a continuous function $f: I \rightarrow R$ which obeys $f\left(t_{n}\right)=x_{n}$ for $n=0,1,2, \ldots, N$.

The previous function is called a Fractal Interpolation Function (FIF) corresponding to $\left\{\left(L_{n}(t), F_{n}(t, x)\right)\right\}_{n=1, \ldots, N^{*}}$

Let $G$ be the set of continuous functions $f:\left[t_{0}, t_{N}\right] \rightarrow[c, d]$ such that $f\left(t_{0}\right)=x_{0} ; f\left(t_{N}\right)=x_{N}$.

$G$ is a complete metric space respect to the uniform norm. Define a mapping $T: G \rightarrow G$ by:

$$
\begin{aligned}
& (T f)(t)=F_{n}\left(L_{n}^{-1}(t), f \circ L_{n}^{-1}(t)\right) \\
& \forall t \in\left[t_{n-1},{ }_{n}\right], n=1,2, \ldots, N .
\end{aligned}
$$

$T$ is a contraction mapping on the metric space $\left(G,\|.\|_{\infty}\right)$

$$
\|T f-T g\|_{\infty} \leq|\alpha|_{\infty}\|f-g\|_{\infty}
$$

where $|\alpha|_{\infty=} \max \left\{\left|\alpha_{n}\right| ; n=1,2, \ldots, N\right\}$. Since $|\alpha|_{\infty}<1, T$ possesses a unique fixed point on $G$, that is to say, there is $f \in G$ such that $(T f)(t)=f(t) \forall t \in$ $\left[t_{0}, t_{N}\right]$. This function is the FIF corresponding to $w_{n}$ and it is the unique $f \in G$ satisfying the functional equation $[5,6]$ :

$$
\begin{aligned}
& f(t)=F_{n}\left(L_{n}^{-1}(t), f \circ L_{n}^{-1}(t)\right) \\
& n=1,2, \ldots, N, t \in I_{n}=\left[t_{n-1}, t_{n}\right], \text { that is to say } \\
& f(t)=\alpha_{n} f \circ L_{n}^{-1}(t)+q_{n} \circ L_{n}^{-1}(t)
\end{aligned}
$$

The most widely studied fractal interpolation

functions so far are defined by the IFS

$$
\left\{\begin{array}{c}
L_{n}(t)=a_{n} t+b_{n} \\
F_{n}(t, x)=\alpha_{n} x+q_{n}(t)
\end{array}\right\}
$$

with

$$
a_{n}=\frac{\left(t_{n}-t_{n-1}\right)}{\left(t_{N}-t_{0}\right)} \text { and } b_{n}=\frac{\left(t_{N} t_{n-1}-t_{0} t_{n}\right)}{\left(t_{N}-t_{0}\right)}
$$

$\alpha_{n}$ is called a vertical scaling factor of the transformation $w_{n}$ and $\alpha=\left(\alpha_{l}\right.$, $\left.\alpha_{1}, \ldots, \alpha_{N}\right)$ is the scale vector of the IFS. If $q_{n}(t)$ is a line, the FIF is termed affine Figure 2 . In this case, by (3) $q_{n}(t)=q_{n 1} t+q_{n 0}$, with:

$$
\begin{aligned}
& q_{n 1}=\frac{x_{n}-x_{n-1}}{t_{N}-t_{0}}-\alpha_{n} \frac{x_{N}-x_{0}}{t_{N}-t_{0}} \\
& q_{n 0}=\frac{t_{N} x_{n-1}-t_{0} x_{n}}{t_{N}-t_{0}}-\alpha_{n} \frac{t_{N} x_{0}-t_{0} x_{N}}{t_{N}-t_{0}}
\end{aligned}
$$

\section{Computation of scaling factors}

Let $\left\{\left(t_{n}, x_{n}\right) ; n=0, \ldots, N\right\}$ be a subset of the data, that here we consider equidistant, $t_{n}=t_{0}+n h$. That values are used as interpolation nodes, and we consider some intermediate points of the signal $t^{j} \in I_{n}=\left[t_{n-1}, t_{n}\right]$, $j=1,2, \ldots, m-1$ as target points to define the fit. If $t$ are also equidistant:

$$
\begin{aligned}
& t^{j}=\frac{(m-j) t_{n-1}+j t_{n}}{m} \\
& L_{n}^{-1}\left(t^{j}\right)=\frac{(m-j) t_{0}+j t_{N}}{m}
\end{aligned}
$$

The value of the FIF at the point $t^{j}$ is given by the equation (6). Replacing the value of the function $f$ at $L_{n}^{-1}\left(t^{j}\right)$ by the value of the polygonal $f_{0}$ (with interpolation nodes $t_{n}$ ):

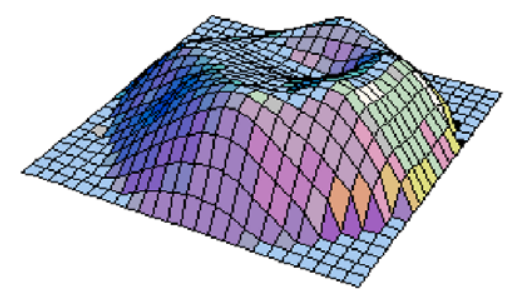

Figure 2: 3D representation of the complexity of an individual executing a visual test. The closest side corresponds to the occipital area. 
$f\left(t^{j}\right) \cong \alpha_{n} f_{0} \circ L_{n}^{-1}\left(t^{j}\right)+q_{n} \circ L_{n}^{-1}\left(t^{j}\right)$

By (9), (10) and (11)

$q_{n} \circ L_{n}^{-1}\left(t^{j}\right)=\frac{(m-j) x_{n-1}+j x_{n}}{m}-\alpha_{n} \frac{(m-j) x_{0}+j x_{N}}{m}$

Therefore, following (12) and (11),

$$
\begin{aligned}
& f\left(t^{j}\right) \cong \alpha_{n}\left(f_{0}\left(\frac{(m-j) t_{0}+j t_{N}}{m}\right)-\frac{(m-j) x_{0}+j x_{N}}{m}\right)+ \\
& +\frac{(m-j) x_{n-1}+j x_{n}}{m} \\
& x^{j}=f\left(t^{j}\right) \cong \alpha_{n} u(j)+v_{n}^{1}(j)
\end{aligned}
$$

Now we compute $\alpha_{n}$ by means of least squares approximation:

$$
\min E\left(\alpha_{n}\right)=\sum_{j=1}^{m-1}\left(\alpha_{n} u(j)+v_{n}^{1}(j)-x^{j}\right)^{2}
$$

In this way, the following value of ${ }_{n}$ is obtained:

$$
\alpha_{n}=\frac{-\sum_{j=1}^{m-1} v_{n}(j) u(j)}{\sum_{j=1}^{m-1} u(j)^{2}}
$$

where

$$
\begin{aligned}
& u(j)=f_{0}\left(\frac{(m-j) t_{0}+j t_{N}}{m}\right)-\frac{(m-j) x_{0}+j x_{N}}{m} \\
& -v_{n}(j)=x^{j}-\frac{(m-j) x_{n-1}+j x_{n}}{m}
\end{aligned}
$$

If $h=t_{n}-t_{n-1}$ tends to zero,

$$
v_{n}(j)=\frac{(m-j) x_{n-1}+j x_{n}}{m}-x^{j}
$$

goes to zero. As a consequence, $\alpha \rightarrow 0$ if $h \rightarrow 0$. This fact allows us to obtain $h$ low enough to get $\left.\alpha\right|_{\infty}<1$.

Bounds of interpolation error and study of convergence are treated in the reference [9].

\section{Fractal dimension}

The first step is the reconstruction of the signal by means of fractal interpolation functions, computing the parameters of the IFS associated to the data according to the fit proposed in the previous paragraph. The computation of the fractal dimension is then performed by the use of explicit formulas.

Following some theorems concerning Iterated Function Systems $[6,10]$, the fractal dimension $D$ of the graph of an affine FIF verifies the equation:

$$
\sum_{n=1}^{N}\left|\alpha_{n}\right| a_{n}^{D-1}=1
$$

where $\alpha_{n}$ is the scaling vertical factor of the IFS and $a_{n}$ the coefficients defined in (8). If the nodes are equidistant, $a_{n}=1 / N$ and

$$
D=1+\frac{\log \left(\sum_{n=1}^{N}\left|\alpha_{n}\right|\right)}{\log N}
$$

This formula for the dimension is valid in the case $1<\Sigma\left|\alpha_{n}\right|$. Otherwise, the fractal dimension is one [6]. This parameter lies between 1 and 2.

\section{Experiment}

Patients: The procedures described were applied to the study of the EEG recordings of two samples of children: a healthy control group and a set diagnosed with an Attention Deficit with Hyperactivity Disorder (ADHD). The clinical manifestations of the ADHD are characterised by a lack of attention, impulsive cognitive and behaviour styles and by an excessive motor activity. Its incidence is estimated between 3 and $5 \%$ of the school population and one or two children with deficient attention per classroom during the first school years may be observed. By a mere visual inspection of the EEG, no difference was observed in the patient group.

Former ADHD EEG studies show the existence of an excessive slow activity, mainly localized in the frontal zone. The differences found in this area aim at a predominant role of the frontal lobe in the study of the attention. In fact, some authors report similarities between the ADHD children and the patients suffering some kind of injury in this region. Both pathologies become apparent by problems of attention and control of impulses, leading to unsuitable behaviour.

The children of control group were selected randomly by the teachers and belong to the same school groups than the children with ADHD. 19 children diagnosed with ADHD were chosen, with an average age of 9.3 and a standard deviation of 1.5. The sample was compared with a control group of 13 children with similar age (9.2) and standard deviation (1.3).

For every subject, the following signals were recorded: (i) an EEG of rest with closed eyes, (ii) an EEG during the execution of a test consisting in the recognition of a face different from the others, in series of three.

Six locations of the cortical surface were analyzed, following the 1020 International System of Jasper: F3, F4, O1, O2, F7, F8. This method was developed to ensure standardized reproducibility so that a subject's studies could be compared over time and subjects could be compared to each other. The " 10 " and " 20 " refer to the fact that the actual distances between adjacent electrodes are either $10 \%$ or $20 \%$ of the total frontback or right-left distance of the skull. Each site has a letter to identify the lobe and a number to identify the hemisphere location. In our case the letters $\mathrm{F}$ and $\mathrm{O}$ stand for frontal and occipital lobes, respectively. Even numbers refer to electrode positions on the right hemisphere, whereas odd numbers refer to those on the left hemisphere. The recording of the signal was performed by an electroencephalograph Grass, connected to the program Rhythm, version 5. The equipment included filters of 0.18 $\mathrm{Hz}$ for low frequencies and $35 \mathrm{~Hz}$ for high frequencies. The sensibility is 7 microvolts per millimeter. The sampling frequency was $128 \mathrm{~Hz}$. A segment of 30 seconds was analyzed inside the second minute.

The fractal dimension of the EEG was obtained by the method proposed, with one intermediate point between every pair of nodes used for the fractal interpolation ( $N$ even, $m=2, j=1)$.

To compare the EEG of rest with the EEG recorded while the execution of the exercise described, the test of the sign hierarchized of Wilcoxon was used. To compare both groups during the execution of the same task the test of Mann-Withney was performed.

Results and discussion: Table 1 shows the average values of the fractal dimension for each group, EEG and channel. This parameter 
Citation: Navascués MA, Sebastián MV, Valdizán JR (2015) Fractal and Smooth Complexities in Electroencephalographic Processing. J Appl Computat Math 4: 198. doi:10.4172/2168-9679.1000198

\begin{tabular}{|c|c|c|c|c|}
\hline & \multicolumn{3}{|c|}{ Control } & \\
\hline & Rest & Test & Rest & Test \\
\hline F3 & 1.71192 & 1.70421 & 1.71802 & 1.74348 \\
\hline F4 & 1.70788 & 1.76038 & 1.70499 & 1.72433 \\
\hline 01 & 1.72900 & 1.75976 & 1.71959 & 1.77173 \\
\hline 02 & 1.74617 & 1.72834 & 1.70886 & 1.77333 \\
\hline F7 & 1.74366 & 1.72131 & 1.69128 & 1.73455 \\
\hline F8 & 1.74458 & 1.73599 & 1.66085 & 1.73310 \\
\hline
\end{tabular}

Table 1: Average values of the fractal dimension for each group, EEG and channel.

undergoes a general increasing on the whole cortical surface by the execution of the visual test in the ADHD group, but the difference is only significant in some locations.

In the comparison of the data obtained in the computation of the fractal dimension of the EEG during the test of faces recognition with respect to the rest EEG, in the group of children with ADHD the differences were found in $\mathrm{O} 2$ to the significance level of 0.05 , and in F8 at level 0.01, whereas in the control group no difference was found. These variations show the activation of the occipital zone (primary visual area) in the achievement of tasks of visual attention, and an increase in frontal area, responsible for cognitive processes. The need of the children with ADHD to activate more cortical networks to perform the same test may be evident.

The results described aim at a lower dimensionality in the resting EEG in general. This fact agrees with the studies of several authors [3] for different pathologies and brain processes.

Some differences between the group of children diagnosed with ADHD and the healthy control at rest were also found. In F8 at the significance level 0.05 and 0.01 , ADHD children show a lower value of the parameter, what confirms the hypothesis of slower resting EEG activity.

\section{Smooth Complexities}

In the present section, we deduce spline formulae for the computation of smooth complexities of any order.

In the early seventies B. Hjorth defined a set of electroencephalographic quantifiers (slope descriptors) which describe the EEG signal and its derivatives in the spectral and time domains.

Several groups have employed these parameters for the EEG processing. To mention some examples, it is worth emphasizing the work of Elbert et al. [11], which use this procedure to evaluate the EEG of a sample of schizophrenic patients. Mouzé-Amady and Horwat [12] compare the results obtained by means of normalized slope descriptors with a conventional FFT analysis on EMG signals recorded from a group of adult subjects while they were executing a manual task of repetitive type. Byring et al. [13] perform a quantitative electroencephalographic analysis of children with learning problems. Ziller et al. [14] carry out a comparative study between Hjorth and nonlinear parameters by means of factor analysis.

In the present study, quadrature formulae for the computation of Hjorth parameters of arbitrary order are proposed. The expressions for the descriptors are obtained by means of an interpolation of the signal by polynomial splines.

To illustrate the procedure, an application to the electroencephalographic study of the Attention Deficit with Hyperactivity Disorder (ADHD) is described.

\section{Slope descriptors}

Let $x(t)$ and $\hat{x}(w)$ be, respectively, the representation of an EEG signal in the time and frequency (Fourier Transform) domains. The spectral moment of order $q$ is defined as

$$
m_{q}=\int_{R} w^{q} S(w) d w
$$

Where $S(w)=\hat{x}(w) \hat{x}(w)^{*}$, and $\hat{x}(w)^{*}$ is the complex conjugate of $\hat{x}(w)$. These moments can be expressed as:

$$
\begin{aligned}
& m_{0}=(1 / T) \int_{I} x(t)^{2} d t=\|x\|^{2} \\
& m_{2}=(1 / T) \int_{I} x^{\prime}(t)^{2} d t=\|x\|_{1}^{2} \\
& m_{4}=(1 / T) \int_{I} x^{\prime \prime}(t)^{2} d t=\|x\|_{2}^{2}
\end{aligned}
$$

The slope descriptors are defined in terms of the moments as:

$$
\begin{aligned}
& A=m_{0} \\
& M=\left(m_{2} / m_{0}\right)^{1 / 2} \\
& C=\left(m_{4} / m_{2}\right)^{1 / 2} /\left(m_{2} / m_{0}\right)^{1 / 2} \\
& C_{q}=\left(\left(m_{2(q+1)} / m_{2 q}\right)-\left(m_{2 q} / m_{2(q-1)}\right)\right)^{1 / 2} \forall q 1
\end{aligned}
$$

$A$ is called activity, $M$ is the mobility and $C$ the complexity. $C$ is the complexity of order $q$. For large $q$, the computation of $m_{2 q}$ implies a numerical differentiation of high order followed by a numerical integration, and this process may produce considerable computation errors. A method of interpolation by polynomial splines is used to reconstruct the signal, and from there the quadratures for $m_{2 q}$ are deduced.

\section{Formulae for the moments}

Let $K^{q}=K^{q}[0, T], q \geq 1(q \in \mathrm{I})$ be the space of real functions defined in the interval $[0, T]$, having a derivative of order $(q-1)$ absolutely continuous and derivative of order $q$ with integrable square. $K^{q}$ is a Hilbert space with the inner product

$$
<f, g>=\sum_{i=0}^{q} \int_{0}^{T} f^{(i)}(t) g^{(i)}(t) d t
$$

Let $\left\{\left(t_{i}, x_{i}\right), i=1,2, \ldots N\right\}\left(t_{i}<t_{i+1} \forall i\right)$ be the set of interpolation points or samples of the signal. Setting $q \geq 1(q \in \mathrm{I})$, let $S$ be the space of real functions $\sigma$, called splines of degree $2 q-1$, defined in $[0, T]$ such that $N-1$

$-\sigma$ is a polynomial of degree $2 q-1$ in each interval $\left(t_{i}, t_{i+1}\right), i=1, \ldots$,

$$
\begin{aligned}
& \text { - } \sigma \text { is a polynomial of degree } q-1 \text { en }\left[0, t_{1}\right) \text { and }\left(t_{N}, T\right] \\
& -\sigma^{(2 q-2)} \text { is continuous. }
\end{aligned}
$$

$S$ is a subspace of $K^{q}$. All $\sigma \in S$ can be expressed as [15]

$$
\sigma(t)=\sum_{j=0}^{q-1} \alpha_{j} t^{j}+\sum_{i=1}^{N} d_{i} \frac{\left(t-t_{i}\right)_{+}^{2 q-1}}{(2 q-1) !}
$$

with

$$
\begin{aligned}
& \left(t-t_{i}\right)_{+}^{2 q-1}=\left\{\begin{array}{lll}
\left(t-t_{i}\right)^{2 q-1} & \text { si } & t \geq t_{i} \\
0 & \text { si } & t<t_{i}
\end{array}\right\} \\
& d_{i}=\sigma^{(2 q-1)}\left(t_{i}+\right)-\sigma^{(2 q-1)}\left(t_{i}-\right)
\end{aligned}
$$

where 


$$
f\left(t_{i}+\right)=\lim _{t \rightarrow t_{i}+} f(t)
$$

and analogously for the other side.

The coefficients $d_{i}$ satisfy the conditions:

$$
\sum_{i=1}^{N} d_{i}\left(t_{i}\right)^{K}=0, \quad K=0, \ldots, q-1
$$

The above equalities along with the interpolation conditions $\sigma\left(t_{i}\right)=x_{i} \forall i=1,2, \ldots N$ allow us to find the coefficients $\alpha_{j}, d_{j}$ and obtain the polynominal spline of interpolation. This function represents the continuous EEG signal, and so it is possible to compute the moments which are necessary to obtain the parameters. The first moments $m_{0}$, $m_{2}, m_{4}$ can be computed by means of cubic splines as these interpolants approximate up to the second derivative of the signal [15]. The higherorder parameters can be obtained by using general polynomial splines of odd degree following the next development.

One of the properties that satisfy the spline functions is [16]:

Theorem 1: $\forall f \in K^{q}$ and all $\sigma \in S$ the following equality holds

$$
\int_{0}^{T} \sigma^{(q)}(t) f^{(q)}(t) d t=(-1)^{q} \sum_{i=1}^{N} d_{i} f\left(t_{i}\right)
$$

In particular, for $f=\sigma$ :

$$
\int_{0}^{T}\left(\sigma^{(q)}(t)\right)^{2} d t=(-1)^{q} \sum_{i=1}^{N} d_{i} \sigma\left(t_{i}\right)
$$

As $\sigma$ is an interpolation spline $\sigma\left(t_{i}\right)=x_{i}$ and so:

$$
\int_{0}^{T}\left(\sigma^{(q)}(t)\right)^{2} d t=(-1)^{q} \sum_{i=1}^{N} d_{i} x_{i}
$$

As a consequence, the following quadrature formula for the moment $m_{2 q}$ is obtained

$$
m_{2 q}=\frac{1}{T} \int_{0}^{T}\left(\sigma^{(q)}(t)\right)^{2} d t=\frac{1}{T}\left[(-1)^{q} \sum_{i=1}^{N} d_{i} x_{i}\right]
$$

\section{Experiment}

We applied the previous method for the EEG study of ADHD. The mobility is considered an index of the mean frequency of the EEG. It is well known that the frequency increases with age and so we have chosen the mobility as a suitable quantifier of the brain maturity.

The mobility was computed for the EEG study of the same samples of children (Section II). The channels processed were F3, F4, P3, P4, $\mathrm{O} 1, \mathrm{O} 2$. The EEG was performed at rest with closed eyes.

Table 2 gathers the mean values of the mobility obtained on different cortical locations for control and patient groups. It can be observed that, in general, the ADHD group presents lower values of the descriptor. These variations have been confirmed statistically by means of a non-parametric test (Mann-Whitney). Significant differences were

\begin{tabular}{|c|c|c|}
\hline & Control & Def At \\
\hline F3 & 47.8627 & 43.3780 \\
\hline F4 & 50.9519 & 44.1308 \\
\hline P3 & 53.8897 & 50.2233 \\
\hline P4 & 50.6928 & 48.9992 \\
\hline O2 & 58.1689 & 51.5804 \\
\hline
\end{tabular}

Table 2: Mean values of the mobility for every group and location. found in the frontal area, locations F3 and F4, for a significance level of 0.05 .

Concerning the ADHD syndrome, the results obtained by other groups indicate the existence of excessive slow activity. The experiments of Mouzé-Amady and Horwat [12] indicate that the mobility is significantly correlated with the mean frequency of the EEG, calculated by means of Fast Fourier Transform. According to these results, children with deficient attention present lower values of the mobility, that is to say, a "slowness" in the EEG with respect to the healthy children, which confirms the described findings.

\section{Current and Future Developments}

We expose in the following items some questions deserving (in our opinion) a special attention in the future. It would be of interest:

- The improvement of the numerical procedures for the calculus of the indices described, in order to reduce the computational time and cost.

- The setting of reliable EEG data bases, including significant parameters of quantification. This fact would help the evaluation of the "normality" of a recording, from a numerical point of view. This matter is specially important in the infantile case, since the child is in a period of maturation and the variability is higher than in the adult case.

- The implementation of multichannel indices, analogous or different to those described in the present article.

- The analysis of the respective relations between the different types of parameters, along with the election of the most suitable quantifiers (or their combinations) in order to describe specific mental processes and pathologies.

\section{References}

1. Mayer-Kress G (1986) Dimensions and Entropies in Chaotic Systems, Springer Verlag.

2. Smith LA (1988) Intrinsic limits on dimension calculations, Phys. Lett. A 133 283-288.

3. Basar E, Basar-Eroglu C, Roschke J, Shultz J (1989) Models of Brain Function, Ed. R.M.J. Cotterill. Cambridge Univ. Press.

4. Lopes da Silva FH, Kamphuis W, Van Neerven JMAM, Pijn JPM (1990) in Machinery of Mind, Ed. E.R. John, Birkhauser.

5. Barnsley MF (1986) Fractal functions and interpolation Constr. Approx. 2: 303329.

6. Barnsley MF (1988) Fractals Everywhere. Academic Press, Inc.

7. Navascués MA, Sebastián MV (2003) Some results of convergence of cubic spline fractal interpolation functions", Fractals 11: 1-7.

8. Navascués MA, Sebastián MV (2004) Fitting curves by fractal interpolation: an application to the quantification of cognitive brain processes. In: Thinking in Patterns: Fractals and Related Phenomena in Nature, Novak MM. Ed., World Sci $143-154$

9. Navascués MA, Sebastián MV (2007) Construction of affine fractal functions close to classical interpolants", Journal of Comp. Analysis and Appl 9: 271-285.

10. Besicovitch AS, Ursell HD (1937) Sets of fraccional dimensions (V): On dimensional numbers of some continuous curves. $J$ of London Math. Soc 12: $18-25$.

11. Elbert T, Lutzenberger W, Rockstroh B, Berg P, Soothsayer R (1992) "Physical aspects of the EEG in schizophrenics". Biol. Psychiatry 32: 595-606.

12. Mouzé-Amady M, Horwat $F$ (1996) Evaluation of Hjorth parameters in forearm surface EMG analysis during an occupational repetitive task", Electroencephalogr Clin. Neurophysiol 101: 181-183. 
Citation: Navascués MA, Sebastián MV, Valdizán JR (2015) Fractal and Smooth Complexities in Electroencephalographic Processing. J Appl Computat Math 4: 198. doi:10.4172/2168-9679.1000198

Page 6 of 6

13. Byring RF, Salami TK, Sainio KO, Örn HP (1991) EEG in children with spelling disabilities". Electroencephalogr Clin Neurophysiol 79: 247-255.

14. Ziller M, Frick K, Herrmann WM, Kubicki S, Spieweg I (1995) "Bivariate global frequency analysis versus chaos theory. A comparison for sleep eeg data". Neuropsychobiol 32: 45-51.
15. Laurent PJ (1972) Approximation et optimisation. Collection Enseignement des Sciences 13. Hermann.

16. Ahlberg JH, Nilson IN, Walsh JL (1967) The Theory of Splines and Their Applications. Academic Press. 